\title{
A Proposed Framework for Implementing Lean Six Sigma Methodology in Ecuadorian Children Hospital
}

Marcos Buestan, Ph.D., Cinthia Perez, Ph.D., Edwin Desintonio, MSc.

Escuela Superior Politecnica del Litoral, Ecuador, mbuestan@espol.edu.ec, edesinto@espol.edu.ec Escuela Superior

Politecnica del Litoral, Ecuador, ccperez@espol.edu.ec

\begin{abstract}
The success of the implementation of Lean
Methodologies and Six Sigma tools has been very well documented, particularly for manufacturing plants. Furthermore, the use of both methodologies, simultaneously, is recommended in scenarios where simplicity and a focus on waste reduction are required. With the objective of taking most of the benefit of the Lean and Six Sigma methodologies, we are interested in designing a framework for the implementation of a Lean Six Sigma (LSS) methodology in an Ecuadorian Children Hospital, where the final goal is to be recognized as a world-class healthcare institution.
\end{abstract}

Keywords - Lean, Six Sigma, Healthcare, Hospital

Digital Object Identifier

(DOI):http://dx.doi.org/10.18687/LACCEI2016.1.1.213

ISBN: 978-0-9822896-9-3

ISSN: 2414-6390

$14^{\text {th }}$ LACCEI International Multi-Conference for Engineering, Education, and Technology: "Engineering Innovations for Global Sustainability", 20-22 July 2016, San José, Costa Rica. 


\title{
A Proposed Framework for Implementing Lean Six Sigma Methodology in Ecuadorian Children Hospital
}

\author{
Marcos Buestan, Ph.D., Cinthia Perez, Ph.D., Edwin Desintonio, MSc. \\ Escuela Superior Politecnica del Litoral, Ecuador, mbuestan@espol.edu.ec, edesinto@espol.edu.ec \\ Escuela Superior Politecnica del Litoral,Ecuador,ccperez@espol.edu.ec
}

\begin{abstract}
The success of the implementation of Lean Methodologies and Six Sigma tools has been very well documented, particularly for manufacturing plants. Furthermore, the use of both methodologies, simultaneously, is recommended in scenarios where simplicity and a focus on waste reduction are required. With the objective of taking most of the benefit of the Lean and Six Sigma methodologies, we are interested in designing a framework for the implementation of a Lean Six Sigma (LSS) methodology in an Ecuadorian Children Hospital, where the final goal is to be recognized as a world-class healthcare institution.

Keywords-Lean, Six Sigma, Healthcare, Hospital
\end{abstract}

\section{INTRODUCTION}

Since the birth of the modern quality philosophies at the mid-twenties, numerous methodologies have proven to be influential in enhancing the quality of manufacturing processes across the world. However, they have never appeared so close to the people as when they have been applied to enhance quality in healthcare systems.

Benefits such as reducing mortality rates in a pediatric Emergency Room (ER), and minimizing the average waiting time in an Emergency Room (ER), show the social impact of continuous improvement projects on healthcare systems.

The efficacy of continuous improvement methodologies has been extensively verified in the literature. Among recent examples of Lean and Six Sigma applications, we have the work presented by Levtzow and Willis [1] that illustrates how Six Sigma principles can reduce laboratory billing errors. Also, Toledo, Carroll, et al. [2] show how the median length of stay for liver transplant patients was reduced by almost $30 \%$ by using Lean Six Sigma methods.

Other authors present frameworks or methodologies applied for implementing continuous improvement initiatives. For instance, Varkey and Kollengode [3] discuss a framework for the implementation of quality improvement (QI) initiatives based on the Plan-Do-Check-Act (PDCA) cycle in healthcare institutions across India. Similarly, Zimmerman, Smith, et al. [4] present three different approaches applied in Emergency Departments (ED) combining Juran's model with the PDCA cycle and the Langley model.

This article is aimed to propose an approach that combine Lean Principles and Six Sigma methodology (LSS) according to the requirements of a Children Hospital located in Ecuador that has recently begun a QI program.

With our methodology, we expect to obtain the fundamental benefits of each approach. Through Six Sigma we will use a group of tools focused on reducing variation that assures the compliance of critical specifications [5], and, the application of Lean principles will allow us to focus on eliminating non-value added steps and removing waste of any form.

Considering task complexity, cultural change at healthcare systems has been a widely studied topic in several articles. For instance, Adrian [6] presents how Lean principles have improved the organizational culture by making employees and physicians observe and see things through patients eyes. According to Arthur [7], the cultural change is not only related to the top management commitment, but also to the informal leaders that act as the hubs in the culture's networks.

Haenke and Stichler [8] show evidence regarding the importance of informal leaders in a case study where transformational nurse leaders were critical in design decisions to improve work and patient care environments in a postanesthesia care unit.

The successful implementation of LSS will not be possible without the engagement and participation of physicians and other healthcare staff [9]. Therefore, our methodology is supported by the development of cultural change that allows employees to be part of the solutions aimed to reduce variation and improve value.

\section{LEAn Six Sigma (LSS) COMbinAtion. A Literature REVIEW}

The combination of Lean and Six Sigma (LSS) methodologies have been remarked in several publications because together, they provide the necessary tools to achieve improvements with formidable results even significantly superior that the expected if they were applied alone [10]. In fact, authors such as Harrison [11] considers that applying Lean and Six Sigma disjointedly is not efficient, because of the formation of sub-cultures for each methodology that could be fighting for the same resources.

Koning, Verver, et al. [12] highlight the benefits of combining Lean and Six Sigma methodologies. According to the authors, Lean offers a system approach, but can be considered short on details, organizational structures or tools. These deficiencies are compensated by the Six Sigma methodology that offers fewer standard solutions but proposes an analytic framework. Precisely, the Six Sigma methodology improves the process effectiveness by using rigorous statistical tools, to uncover the roots of problems and, therefore, the reduction or elimination of the sources of variability.

Besides, there is practical evidence that shows that Six Sigma alone is not enough Harrison [11]. Under the Six 
Sigma methodology, improvement projects can take months to get significant results because the initiative is launched by Black Belts (BBs), who are isolated from the shop floor, and may develop initiatives that are not necessarily related to the company strategy.

Under this scenario, the application of an agile methodology like Lean can be used to speed up quality improvement processes during a Six Sigma implementation. Lean offers to employees the necessary understanding to take forward improvement initiatives and identify the processes that could be leveraged by Six Sigma [13].

Several approaches have been suggested for implementing the LSS methodology. Most of them have been developed under the Define, Measure, Analyze, Improve and Control (DMAIC) methodology, an extension of the Plan-DoCheck-Act (PDCA) cycle, like the proposed by Yeh, Lin et. al [14]. According to Snee (2010) even today the DMAIC could be considered the best available approach for LSS implementation because of its elegance and simplicity [8]. Montgomery [15] states DMAIC as the traditional framework to achieve operational excellence [6].

Mousa [16] considers in his approach an eight phases framework called Select-Define-Measure-Value StreamImprove-Control-Replication (SDMVAICR). In this approach, additionally to the classic DMAIC, Mousa proposes a Select, a Value Stream Mapping and a Replication phase. This phase focuses specifically on the project selection process. Furthermore, a Value Stream Mapping (VSM) stage is incorporated after the Measure phase. VSM is aimed to identify the root causes of waste and the application of Lean principles. Finally, the Replicate phase aims to identify opportunities alongside the plant, where the solutions established in the Improve phase are suitable to be implemented.

Another approach has been proposed by Thomas et. al. [17] that incorporates Lean and Six Sigma in one framework intended primarily for the application of Design of Experiments (DOE). The proposed framework presents a noticeable difference between the periods of application of both methodologies. In this case, Lean methodology is used to identify areas of waste and performance improvement while Six Sigma tools are used to determine the main effects and interactions of significant factors that affect a specific response variable.

Furterer and Elshennawy [18] report the implementation of total quality management (TQM) and LSS in a local government. They propose a framework that combines the principles of Lean Enterprise and Six Sigma. Their approach is based on the DMAIC cycle approach, in which they include a waste identification stage as part of the Analyze and Improve phases; and suggest the application of a group of Lean principles such as, kanban and visual control, as part of the solution.

Similarly, Kumar, Antony, et al. [19] proposes a LSS framework in a case study that presents its application in an Indian SMEs. Using a DMAIC approach the authors combine both methodologies including VSM technique as part of the
Measure phase to identify improvement opportunities. Additionally, Lean tools such as 5S, Total Productive Maintenance (TPM), or the developing of proofing mistake systems are integrated as part of the Improve or Control phases.

A similar approach proposed by Lee and Wei [20] relates the application of LSS to reduce mold changing time. Their combined methodology adopts Six Sigma and Lean tools simultaneously. According to the DMAIC framework, the authors incorporate Lean tools like VSM as part of the Measure phase and TPM and 5S as initiatives that enhance the effectiveness of the Six Sigma tools during the Improve phase.

The primary difference among the proposed LSS approaches relies in the way that they combined the Lean and Six Sigma methodologies. Assarlind et al. [21] consider that the term Lean Six Sigma can be used as a combined entity representing a full integration of Lean principles and Six Sigma in only one approach, as one initiative. However Assarlind et al. [21] also mention the application of LSS, as the use of the most suitable tools of each methodology, where they are applied simultaneously rather than in full integration.

The application of Lean Six Sigma in Hospitals has been remarked as a suitable methodology in numerous publications. For instance, Curatolo and Lamouri [22] consider that LSS is suitable to healthcare systems based on two main reasons. First, it is an intuitive and compelling methodology that can be easily adapted for healthcare staff, and second, the focus of Lean on eliminating or minimizing every type of waste, which is a recurrent topic for hospitals. Similarly, Mason, Nicolay, et al. [23] after a revision that includes 124 potentially relevant studies, indicate the important role of Lean and Six Sigma in generating significant improvements across a variety of surgery outcomes related to the pre-operative, operative and in-patient settings.

\section{BACKGROUND OF LEAN SIX SIGMA (LSS) IMPLEMENTATION}

Social responsibility is one of the strategic goals at the Escuela Superior Politecnica del Litoral (ESPOL). Given that ESPOL strategy for social responsibility is addressing major local problems, the Industrial Engineering (IE) department proposed a long-term project within an Ecuadorian Children Hospital $(\mathrm{ECH})$ to achieve a world-class status.

The Ecuadorian Children Hospital $(\mathrm{ECH})$ considered in this study, is a private non-profit organization dedicated to help the community since 1906. This hospital is part of a healthcare institutions network in Guayaquil-Ecuador.

Initially, the hospital was a small outpatient health facility, financially supported by a group of philanthropists. Nowadays, the hospital has a capacity of 160 care beds, 5 operating rooms, a unit of intensive care, laboratory, pharmacy and a physical therapy unit. Hospital occupancy is 160 patients on an average day, outpatient service is required by 140 patients on an average day.

During the initial phase of the project, a group of IE students and professors started a preliminary evaluation of the 
ECH processes, with the goal of identifying QI opportunities that later, will be addressed as improvement projects.

The projects were evaluated using an impact-effort matrix that revealed which projects might be considered as a priority for the hospital. In most of the cases, the problems were related to a deficient patient flow and a lack of processes standardization. Given the numerous successful experiences related to the application of LSS in a healthcare environment, and the characteristics of the problems in the ECH, the hospital managers, and the university's consulting group decided to implement LSS.

An important aspect was discovering the right path for the implementation of LSS at the Hospital. We selected an incremental improvement approach. Starting with small steps, instead of implementing a very complex continuous improvement system that could require a deep level of support and a significant use of resources. The selected approach starts with few projects that focused directly on solving problems to improve primary key performance indicators (KPI).

The proposed approach considers the suggestion of authors such as Curatolo and Lamouri et al. [22] that recommend starting a Lean implementation on a micro level. Hence, Lean should be applied to specific operational processes previous the deployment of the Lean principles to the entire organization.

Similarly, Burk [24] during the implementation of the LSS program in the American Navy suggested, to the high command, to start the process with a small portfolio of projects with a short duration and objectives easy to accomplish.

Based on the previous observations, an incremental approach will help to create the confidence in applying the LSS methodology inside the hospital. Additionally, this approach promotes a culture that is comfortable with changes, reducing the resistance to the implementation of new solutions significantly.

\section{Proposed LEAN Six Sigma FrameWORK}

In our proposed approach presented in Figure 1, Lean and Six Sigma are combined in only one initiative. Depending on the expected output, each tool is assigned to different phases. Although DMAIC is maintained as the framework for the inclusion of Lean and Six Sigma tools, our proposed approach presents an alternative framework related to the five Lean principles proposed by Womack and Jones [25] in the classic book "Lean Thinking" namely [10]:

- Identify Value

- Map Value Stream

- Create Flow

- $\quad$ Establish Pull
- Pursue Perfection

Precisely, our proposed approach presented in Figure 1 offers a clear combination of both methodologies. The approach has been developed considering the recommendations offered by Clegg, Pepper, et al. [13] that states four guides during the construction of a Lean Six Sigma approach namely:

- $\quad$ Strategic and process focused

- Balance between the two philosophies

- Balance between complexity and sustainability

- Designed considering the kind of problems experienced.

We take advantage of the characteristic elements of the Lean or Six Sigma methodology that can have a long-term impact on the LSS program ultimate success, such as:

A structured approach: highly defined structure proposed for DMAIC projects integrated by a Champion, Green Belts (GB), Black Belts (BB) and process owner.

Project-based deployment: The establishment of QI using a project-based approach offers to the company an effective scoping and the definition of quantifiable metrics to be improved within a reasonable period of time.

A detailed plan and build low-level improvement team: The establishment of a detailed plan for QI implementations managed by low-level improvement teams allow technical and operative personnel get more in concern with the implementation process. The result is an increment in the probabilities that solutions could be applied by driving the improvement initiatives from the bottom-up [26].

In the following lines, a logical explanation of each of the proposed stages is presented.

Recognize/Identify Value: This primary phase is not associated directly with a specific project. It can be considered a diagnosis stage that offers a project portfolio to the company based on the identification of problems related to an excessive variation at any process step or a deficient flow in the process. Problem areas identification and project selection are the most critical factors to achieve and sustain the desired improvement. An appropriate project selection offers the necessary information to determine the specific tools and the right personnel that should be assigned to achieve the maximum process improvement [27] [8]. 


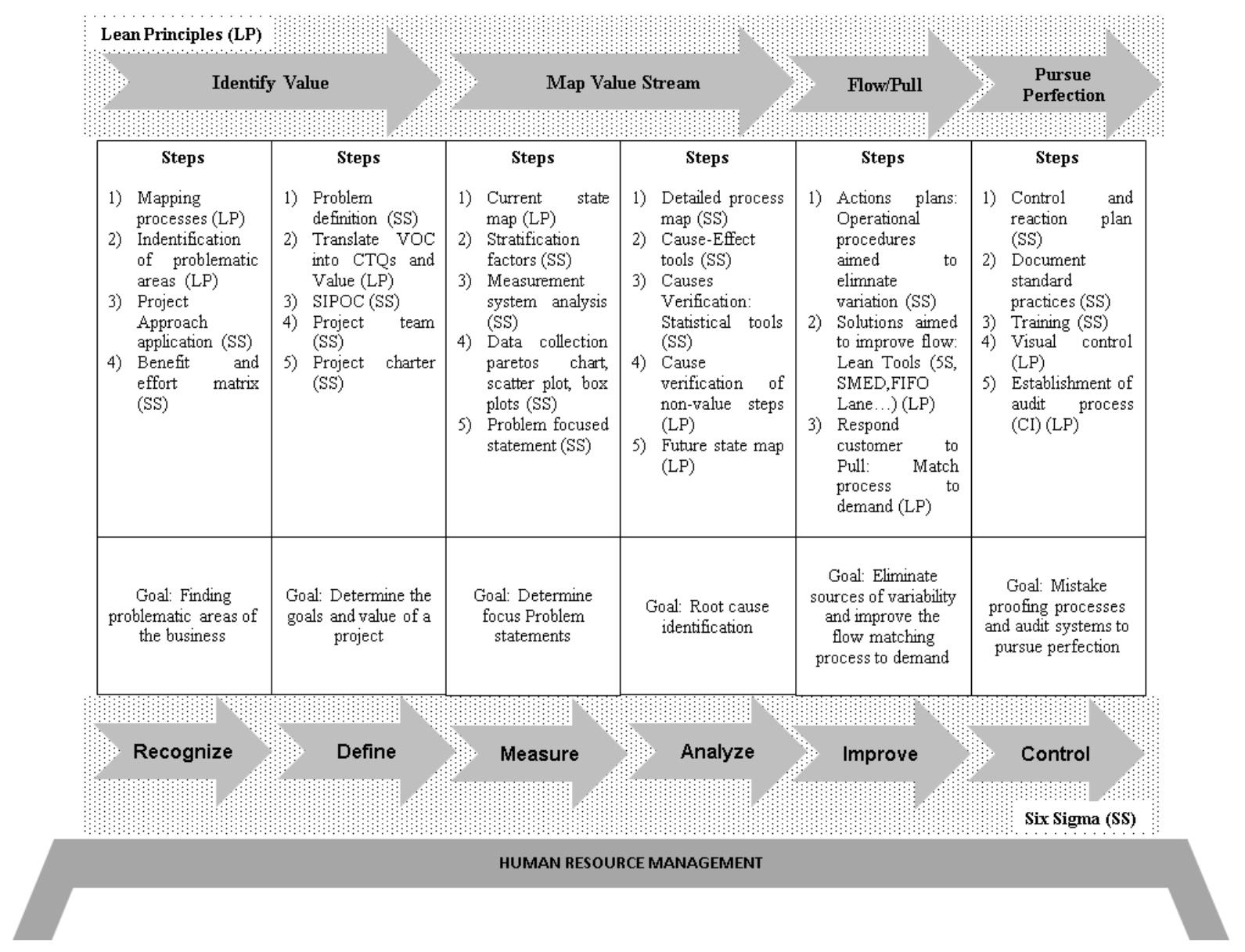

Fig. 1 Proposed Lean Six Sigma Framework.

This phase begins with a mapping of the processes aimed to identify the primary needs to apply the LSS methodology. These needs should be in agreement with the specifications established for each of the hospital management indicators. In this way, the mapping should be developed with the necessary detail to identify improvement opportunities.

Once the opportunities have been identified, they should be combined in such a way that together; they could be considered as one project. With the application of a benefit and effort matrix, it will be possible to evaluate each of the potential initiatives relative to the efforts in terms of the time or resources required.

Define / Identify Value: In this phase the objective is to establish the specific need that has to be solved with the project. A clear definition of the problem is obtained through the application of the $5 \mathrm{~W} 1 \mathrm{H}$ tool: "What, Where, When, Who and How much".

Once the voice of the customer (VOC) is obtained, it is necessary to translate this VOC into critical to quality (CTQs) factors using a CTQ tree. The translation is based on a group of driver factors that make this conversion easy. Besides, the $5 \mathrm{~W} 1 \mathrm{H}$ tool is applied to identify what it is understood as value for the customer.
Then, the scope of the project is established by using a Supplier Input Process Output Customer (SIPOC) diagram. This tool offers the necessary information to define the appropriate members of the project team based on the selected process steps, inputs, and output variables.

Finally, the project charter as a standardized document that state the scope, objectives, and participants of the project is applied as a brief summary of the project deliverables. Additional information such as the expected deliverables, the related KPIs associated with the project, a summary of the schedule and a business case are included in the document.

Measure / Map Value Stream: The primary objective of this phase is to clarify the problem through the establishment of focus problems statements that split the original problem into specific areas. The advantage of this phase relies on in that it is easier to find potential causes for a problem when such a problem is concentrated in one single area. Hence, the more clarified and enclosed is the problem the easier is to determine its potential causes in the subsequent phases.

The measure phase begins developing a VSM of the application of the current state that allows us to identify the primary steps blocking the continuous flow alongside the process. Also, the VSM is used to determine the steps where 
the variability is a problem because the processing is not according to the specifications.

In some cases the problems obtained from the VSM are clear However in other cases, they need to be analyzed, considering a group of selected stratification factors such as time, suppliers or departments.

To collect the required data based on the stratification factors, it is necessary to verify the accuracy and precision of the measurement system. The verification not should be limited to physical equipment but also to the current procedures or administrative steps established for the collection of data.

After the data needed is collected, the data analysis is made according to the stratification factors. Statistical and quality tools such as Pareto Charts, Scatter Plots or Box plots are applied for the analysis.

The focused problems are defined using a variation of the $5 \mathrm{~W} 1 \mathrm{H}$ tool applied in the previous Define phase. At this case, the $4 \mathrm{~W} 1 \mathrm{H}$ include the questions "What, How, When, Where and Who".

Analyze / Map Value Stream: The objective of this phase is to identify and verify the causes related to each of the problems focused statements.

Detailed process maps of the critical areas are a good option to make the identification of potential causes easier and reliable. In fact, it is much more difficult to lose potential causes when a brainstorming is supported with a detailed map. The potential causes are priosied by using a Cause-Efect matrix. The use of verification plans is recommended o avoid developing action plans for trivial causes.

Statistical tools or "Gemba" observation can be used to verify potential causes. Both techniques should be aimed to record the necessary evidence to verify or deny a potential cause of variation or a source of waste. Additional to quantitative data, collected evidence could include another kind of information such as videos, pictures, surveys, or even prints of computer screens.

Causes with solid evidence continue the analysis. Therefore, the 5Whys technique is applied to guarantee all the causes that will be solved in the next phase can be considered root causes.

Finally, based on the verified causes a Future State Map is developed to minimize or eliminate the identified causes of variation or sources of waste.

Improve/Flow/Pull: The objective of the improve phase is to establish and implement actions aimed to minimize or eliminate the root causes of variation and the identified sources of waste. At this phase, an action plan is a management tool that has to be applied to keep track of the improvement actions. Precisely, the lack of control during the implementation phase has been considered one of the primary blockages that affect the success of the methodology negatively.

The proposed solutions range from "Just do it" improvement actions to the applications of other methodologies such as Design of Experiments (DOE) or computer simulations. Note, that the action plan could include pilot test developed in the real environment aimed to determine the effectiveness of the proposed initiatives.

Solutions aimed to improve flow have to be implemented using as a reference the future state map. Doctors, administrative personnel and sanitary staff should be trained in Lean tools such as 5S, Poka-Yoke, Visual control, FIFO lane, among others before solutions are developed.

The Improve phase has to include as a primary requirement the presentation of observable and verifiable results that demonstrate the effectiveness of the proposed initiatives and solutions.

Control/Pursue Perfection: Numerous Six Sigma implementations do not develop this phase aimed to sustain the improvement over the long term. On the contrary, usually, the teams consider the projects closed as immediately as they got the first evidence of improvement. Precisely, this is a serious risk that practitioners and researchers alert as one of the primary factors that jeopardize a Six Sigma or Lean implementation.

The fact is, that the outstanding results found in the Improve phase, usually overshadow the need of implementing procedures that guarantee the long term effectiveness of the solutions. Project leaders believe improvements can be sustained in a natural way without reinforce and control methods. To avoid this issue, the proposed framework incorporates the cultural aspect of Lean, which brings to the projects the characteristic of a continuous journey. In practice, the previous implies the establishment of a formal procedure with activities aimed to prevent the causes of variation and the sources of waste that could reappear.

The aim of standardization is to document the best practices implemented as part of the Improve phase, becoming the baseline for future improvements. So, the application of an appropriate training system is an invaluable tool to maintain a continuous improvement culture. Therefore, the Hospital should develop an appropriate training system that enables employees to learn the skills and maintain the best practices suggested in the improve phase.

The application of Visual Controls and Poka-Yoke systems compliment the previous tools because they use documentation and training to eliminate human errors.

Finally, the establishment of an audit process guarantees that the improvement activities will be maintained in the long term even if the project leader could not be in charge anymore.

It is important to mention that our proposed framework is supported by the inclusion of human resources management elements (HRM) aimed to underpin a cultural change. Several authors report factors like the level of empowerment and education of the people as highly factors related to the workers satisfaction during the implementation of a LSS methodology. In fact, authors such as Tolga Taner, Sezen, et al. [28] state that not considering the psychology of the workforce 
appropriately could appear as an internal barrier for a successful SS implementation in the healthcare.

Other authors like Henderson and Evans [29] state that the keys for a SS successful implementation include factors related to human resources management namely: Upper management support and involvement, training, communication plan, compensations programs, promotion requirements changes and organizational infrastructure.

The previous factors are similar to the presented by Coronado and Antony [30] that additionally include factors such as: Developing project management skills and linking Six Sigma to the customers and the business strategy.

In this way, our suggested approach proposes the inclusion of changes in the culture aimed to promote an environment that break the barriers of poor expectations directly with the participation of the front line employees.

This includes taking advantage of a high-performing idea system that allows getting significant numbers of QI ideas from the people that have the ability to improve their own work environment.

Authors such as Robinson and Schroeder [31] studied 300 organizations in 25 countries, where they identified a relationship between the performance of the companies' frontline ideas systems and their success in the Lean effort.

However, the program success is not related to maintaining actions that appear almost indifferent to the employees. The most popular example is the commonly used "suggestion box".

On the contrary, the focus is to get a large number of improvement ideas that according to Robinson and Schroeder [31] should exceed the 12 or more ideas per employee per year. To obtain this expected result our proposed approach incorporate the following four principles:

- Ideas integrated into the day-to-day work

- The program put the emphasis on small ideas

- Ideas tied to key company improvement goals

- Efficient methods that assure a prompt action on ideas

Establishing the previous principles requires developing fundamental changes that will be integrated into the proposed LSS implementation approach.

Ideas integrated into the day-to-day work: Allowing healthcare employees to offer their ideas voluntarily and unstructured is not efficient. This approach usually result at a poor production of suggestions. Therefore, the proposed approach includes the establishment of formal 30 minutes week meetings around each of the projects developed.

The program put the emphasis on small ideas: Big ideas with a great overall impact could not be easy to implement. Usually, this kind of ideas is related to a major cost that the Hospital could not afford. In this way, the leader's meetings should be prepared to motivate the generation of numerous ideas much more suitable that accumulated can make a difference.
The ideas tied to key company improvement goals: Relating the meetings to the necessary activities for the development of a specific project assure that the collected ideas will be aligned to the most problematic areas.

Well-known experiences appear at the Measure and Analyze phases. At both cases the brainstorm activities occur within the scope of the process and the identified ideas are directly related to the sequence of the events. This is a way of utilizing the brainstorming in a more focused and productive way.

Efficient methods that assure a prompt action on ideas: The sustaining at the long term of a high performing idea system is related to the continuous monitoring and efficient implementation of the proposed ideas.

For this, the application of a project approach could be an appropriate option because of the influence that the team project has over the application of the proposed ideas.

This is supported by the reward system established for the project team members usually related to the finishing of the project within the planning horizon and by the impact of the implemented ideas in the bottom-line results.

\section{DISCUSSION}

The application of continuous improvement methodologies in healthcare environments has proven to produce outstanding results, similar to the ones achieved in manufacturing fields. This kind of results have promoted the appearing of several approaches aimed to combine Lean Principles and Six Sigma methodology's elements in practice.

However, there is a lack of formal methodologies for implementing LSS in healthcare environments. The critical role of the staff and physicians makes necessary the inclusion of a human resource management module that guarantees the success of the methodology.

We propose a Lean Six Sigma framework for healthcare systems that enables the engagement of the staff and promotes their participation.

\section{ACKNOWLEDGMENT}

We would like to thank the valuable help from all of the people at the Children Hospital Leon Becerra who were involved at this research.

We also appreciate the comments received from the anonymous referees.

\section{REFERENCES}

C. B. Levtzow and M. S. Willis, "Reducing Laboratory Billing Defects Using Six Sigma Principles," Laboratory Medicine, vol. 44, pp. 358-371, 2013.

A. Toledo, T. Carroll, E. Arnold, Z. Tulu, T. Caffey, L. Kearns, et al., "Reducing liver transplant length of stay: a Lean Six Sigma approach," Progress in transplantation, vol. 23, pp. 350-364, 2013.

$14^{\text {th }}$ LACCEI International Multi-Conference for Engineering, Education, and Technology: "Engineering Innovations for Global Sustainability”, 20-22 July 2016, San José, Costa Rica. 
[3] P. Varkey and A. Kollengode, "A framework for healthcare quality improvement in India: The time is here and now!," Journal of postgraduate medicine, vol. 57, p. 237, 2011.

[4] R. Zimmerman, R. Smith, C. M. Fernandes, and T. Smith, "A quest for quality," Quality progress, vol. 39, p. 41, 2006.

[5] J. P. Ficalora and L. Cohen, Quality function deployment and Six Sigma: a OFD handbook: Pearson Education, 2009.

[6] N. Adrian, "A lean Transformation," Quality Progress, vol. 44, p. 40, 2011.

[7] J. Arthur, "Seduce them with success," Quality Progress, vol. 38, p. 35,2005 .

[8] R. Haenke and J. F. Stichler, "Applying Lean Six Sigma for Innovative Change to the Post-Anesthesia Care Unit," Journal of Nursing Administration, vol. 45, pp. 185-187, 2015.

[9] C. Caldwell, J. Brexler, and T. Gillem, "Engaging physicians in lean Six Sigma," Quality Progress, vol. 38, p. 42, 2005.

[10] B. Smith, "Lean and Six Sigma-a one-two punch," Quality progress, vol. 36, pp. 37-41, 2003.

[11] J. Harrison, "Six Sigma vs. lean manufacturing: Which is right for your company," Foundry Management \& Technology, vol. 13, pp. 31-32, 2006.

[12] H. Koning, J. P. Verver, J. Heuvel, S. Bisgaard, and R. J. Does, "Lean six sigma in healthcare," Journal for Healthcare Quality, vol. 28, pp. 4-11, 2006.

[13] B. Clegg, M. Pepper, and T. Spedding, "The evolution of lean Six Sigma," International Journal of Quality \& Reliability Management, vol. 27, pp. 138-155, 2010.

[14] H.-L. Yeh, C.-S. Lin, C.-T. Su, and P.-C. Wang, "Applying lean six sigma to improve healthcare: An empirical study," African Journal of Business Management, vol. 5, p. 12356, 2011.

[15] D. C. Montgomery, "A modern framework for achieving enterprise excellence," International Journal of Lean Six Sigma, vol. 1, pp. 56-65, 2010.

[16] A. Mousa, "Lean six sigma Model," International journal of Scientific \& Engineering research, vol. 4, pp. 1154-1162, 2013.

[17] A. Thomas, R. Barton, and C. Chuke-Okafor, "Applying lean six sigma in a small engineering company-a model for change," Journal of Manufacturing Technology Management, vol. 20, pp. 113-129, 2008.

[18] S. Furterer and A. K. Elshennawy, "Implementation of TQM and lean Six Sigma tools in local government: a framework and a case study," Total Quality Management \& Business Excellence, vol. 16, pp. 1179-1191, 2005.

[19] M. Kumar, J. Antony, R. K. Singh, M. K. Tiwari, and D. Perry, "Implementing the Lean Sigma framework in an Indian SME: a case study," Production Planning and Control, vol. 17, pp. $407-$ 423\%@0953-7287, 2006.

[20] K. L. Lee and C. C. Wei, "Reducing mold changing time by implementing Lean Six Sigma," Quality and Reliability Engineering International, vol. 26, pp. 387-395, 2010.

[21] M. Assarlind, I. Gremyr, and K. Bäckman, "Multi-faceted views on a Lean Six Sigma application," International Journal of Quality \& Reliability Management, vol. 30, pp. 387-402, 2013.

[22] N. Curatolo, S. Lamouri, J.-C. Huet, and A. Rieutord, "A critical analysis of Lean approach structuring in hospitals," Business Process Management Journal, vol. 20, pp. 433-454, 2014.

[23] S. Mason, C. Nicolay, and A. Darzi, "The use of Lean and Six Sigma methodologies in surgery: A systematic review," The Surgeon, vol. 13, pp. 91-100, 2015.

[24] M. Burk, "A Six Sigma Program Under Way," in Six Sigma Forum Magazine, 2009.

[25] J. P. Womack and D. T. Jones, Lean thinking: banish waste and create wealth in your corporation: Simon and Schuster, 2010.

[26] S. S. Chakravorty, "Six Sigma programs: An implementation model," International Journal of Production Economics, vol. 119, pp. 1-16, 2009.

[27] R. D. Snee, "Lean Six Sigma-getting better all the time," International Journal of Lean Six Sigma, vol. 1, pp. 9-29, 2010.

[28] M. Tolga Taner, B. Sezen, and J. Antony, "An overview of six sigma applications in healthcare industry," International Journal of health care quality assurance, vol. 20, pp. 329-340, 2007.

[29] K. M. Henderson and J. R. Evans, "Successful implementation of six sigma: benchmarking General Electric Company," Benchmarking: An International Journal, vol. 7, pp. 260-282, 2000.

[30] R. B. Coronado and J. Antony, "Critical success factors for the successful implementation of six sigma projects in organisations," The TQM magazine, vol. 14, pp. 92-99, 2002.

[31] A. G. Robinson and D. M. Schroeder, "The role of front-line ideas in lean performance improvement," The Quality Management Journal, vol. 16, p. 27, 2009.

$14^{\text {th }}$ LACCEI International Multi-Conference for Engineering, Education, and Technology: "Engineering Innovations for Global Sustainability”, 20-22 July 2016, San José, Costa Rica. 